\title{
Performance estimations for the ITER bolometer diagnostic
}

\author{
H. Meister ${ }^{\mathrm{a}}$, F. Penzel ${ }^{\mathrm{a}}$, Z. Szabo-Balint ${ }^{\mathrm{b}}$, R. Reichle ${ }^{\mathrm{d}}$, L.C. Ingesson ${ }^{\mathrm{c}}$ \\ ${ }^{a}$ Max-Planck-Institut für Plasmaphysik, Boltzmannstr. 2 D-85748 Garching, Germany \\ ${ }^{b}$ Wigner Research Centre for Physics, Hungarian Academy of Sciences, Konkoly-Thege Miklós 29-33, H-1121 Budapest, Hungary \\ ${ }^{c}$ Fusion for Energy, c/ Josep Pla, $n^{\circ}$ 2, 08019 Barcelona, Spain \\ ${ }^{d}$ ITER Organisation, Route de Vinon-sur-Verdon, CS 90 046, 13067 St. Paul Lez Durance Cedex, France
}

\begin{abstract}
The ITER bolometer diagnostic shall provide the measurement of the total radiation emitted from the plasma, a part of the overall energy balance. Up to 550 lines-of-sight (LOS) will be installed in ITER observing the whole plasma from many different angles to enable reliable measurements and tomographic reconstructions of the spatially resolved radiation profile. The performance of the diagnostic is intimately linked to the constraints imposed by the design requirements, the sensor and data acquisition design, as well as expected noise levels.

The results presented contain the estimated signal intensities for the current design and integration of bolometer cameras in ITER, based on the simulated radiation profile for the ITER standard scenario (DT-plasma at $15 \mathrm{MA}$, $500 \mathrm{MW}$ with low impurity content). The corresponding power deposited onto the absorber ranges between $0.5 \mu \mathrm{W}$ and $2 \mathrm{~mW}$. The expected noise levels derived mainly from lab measurements and compared to operating bolometer systems yield estimated values for ITER in the order of $1 \mu \mathrm{W}$, clearly indicating a need for optimizing the diagnostic.

The optimization potential is discussed. Cameras for which the light yield could be increased are identified. Also, the potential for enhancing the sensitivity by using different absorber thicknesses is explored.
\end{abstract}

Keywords: ITER, Bolometer, Plasma diagnostics, Performance

\section{Introduction}

The total radiated power as well as the radiation emission profile on ITER will be determined by the bolometer diagnostic. A bolometer measures the plasma radiation over a wide spectral range (from soft-X to the infrared) by monitoring the temperature rise induced by deposition of photon energy in the absorber layer of the bolometer. The reference detector type chosen for ITER is the metal resistor bolometer $[1,2]$. Up to 550 linesof-sight (LOS) will be installed in ITER observing the whole plasma from many different angles to enable reliable measurements and tomographic reconstructions of the spatially resolved radiation profile. The performance of the diagnostic is intimately linked to the constraints imposed by the design requirements, the sensor and data acquisition design, as well as expected noise levels.

This paper describes the current status of the viewing geometry of the ITER bolometer diagnostic and its implications for the performance. Section 2 gives a brief overview of the distribution of LOS and requirements influencing it. Section 3 discusses the estimated signal intensities and compares them to the expected noise levels. Section 4 proposes possibilities to improve the diagnostic performance and Section 5 concludes on future work.

\section{Overview of the ITER bolometer diagnostic}

For an accurate tomographic reconstruction the ITER bolometer diagnostic should provide many LOS covering the plasma cross section from many different viewing angles. Their integration into the vessel is limited by the available construction space. Locations on the vacuum vessel (VV) wall have challenging interfaces with blanket modules (BM) and require dedicated cut-outs for the camera housing as well as a clear view through the gap between BMs. A detailed investigation on this topic is given in [3]. Locations on divertor cassettes need to consider the remote-handling $(\mathrm{RH})$ movements of cassettes during installation which significantly constrict the space envelopes available for bolometer cameras. Locations inside the equatorial port plug (EPP) number 1 (E01) and the upper port plugs (UPP) numbers 1 and 17 (U01 and U17) also have restricted 
space envelopes for their integration because features for attachment of first-wall components and diagnostic shielding modules as well as neighbouring diagnostics strongly limit the available positions for bolometers. In all locations the available number of feedthroughs for the signal lines is another important limiting factor. Finally, according to the project requirements of ITER the spatial resolution in the divertor area shall be $5 \mathrm{~cm}$ and in the rest of the plasma $\frac{a}{30} \approx 6.6 \mathrm{~cm}$, with the minor plasma radius $a$ [4]. As the definition of a spatial resolution for tomographically reconstructed profiles is all but trivial, it was decided to use the average distance between LOS in the observation area as criterion.

The design integration efforts led to 550 individual LOS which are defined by 71 cameras distributed toroidally and poloidally over the ITER VV. It is based on the work performed for the Conceptual Design Review (CDR) following ideas generated already for the first ITER design $[5,6]$. Since the CDR the LOS distribution evolved to account for integration issues. However, the main distribution of the LOS remained. Fig. 1 shows the LOS mapped into one poloidal plane on the left and a schematic of the toroidal distribution on the right. A high density of LOS is gathered within sectors
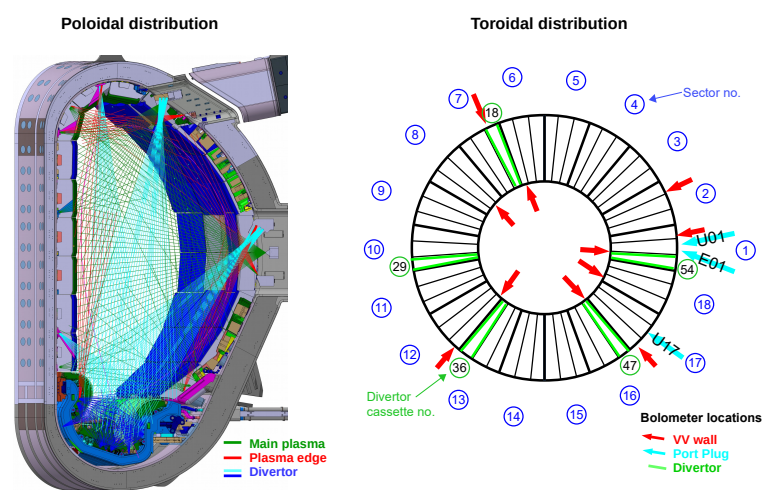

Figure 1: ITER bolometer LOS mapped into one poloidal plane (left) and a schematic of the toroidal distribution (right).

1, 16 and 17 providing the main data for tomographic reconstructions and fulfilling the requirement on spatial resolution. Additionally, LOS in the sectors 7 and 12 provide information on any potential asymmetry in the toroidal distribution of the plasma radiation, which will be used in particular for the optimisation of the disruption mitigation system. A fraction of all LOS doubles some of the views in adjacent toroidal positions and provides a certain level of redundancy. Orienting these LOS slightly different than the original ones helps to enhance the spatial resolution in case all of them are operational. The exact amount of LOS required for re- dundancy still has to be determined after completion of the analysis of the whole system for reliability, accessibility, maintainability and inspectability (RAMI).

The desired viewing geometry of each LOS depends on the foreseen observation area in the plasma cross section, the spatial resolution of the radiation profile to be achieved and the interfaces with adjacent components. It can be defined either by collimators or by pinhole cameras. Methods to derive the main design parameters of these camera types are described in [7] in detail. Their application led to the decision to use collimators for cameras located on the $\mathrm{VV}$ and in the divertor. For port mounted cameras it turned out to be advantageous to combine both, pinholes and collimators within the respective housings. The collimators provide better spatial resolutions for LOS observing the divertor area whereas the pinholes offer a good coverage of the main plasma.

\section{Estimated LOS intensities}

In order to assess the performance of the bolometer diagnostic the expected signal intensities have been estimated using a predicted plasma radiation profile for ITER and the LOS geometries designed according to the restrictions given in Section 2. So far only the radiation profile for the case of a standard 15 MA plasma with low impurity content $(\mathrm{H}$ isotopes as main ion species with $2 \% \mathrm{Be}, 0.01 \% \mathrm{~W}, 0.1 \% \mathrm{Ne}$ and $0.1 \% \mathrm{Ar}$ ) is available, yielding a core radiated power of about $51 \mathrm{MW}$ and radiated power from divertor and plasma edge summing up to $14 \mathrm{MW}$. It has been built from separate SANCO (core) and SOLPS (edge and divertor) simulations by mapping individual results onto a common grid in the poloidal cross section, which is based on the ITER plasma equilibrium for the chosen scenario.

The synthetic signals were calculated for every LOS based on the above given radiation profile and using the routines provided by the ToFu library [8]. The resulting signals span four orders of magnitude and depend on the radiation intensity in the observed region and the étendue of each individual LOS. A summary of the variation is given in Fig. 2. For each generic location the power on the absorber for each LOS is given before and after the re-definition of LOS-geometry which is discussed in Section 4. The power levels also include a factor of 0.5 to account for the effect of a microwave reflecting grid [9], which was not included explicitly in the calculations performed using ToFu.

A first estimation on the expected noise levels for bolometer sensors has been given in [10]. Based on measurements in an ideal environment and assuming 

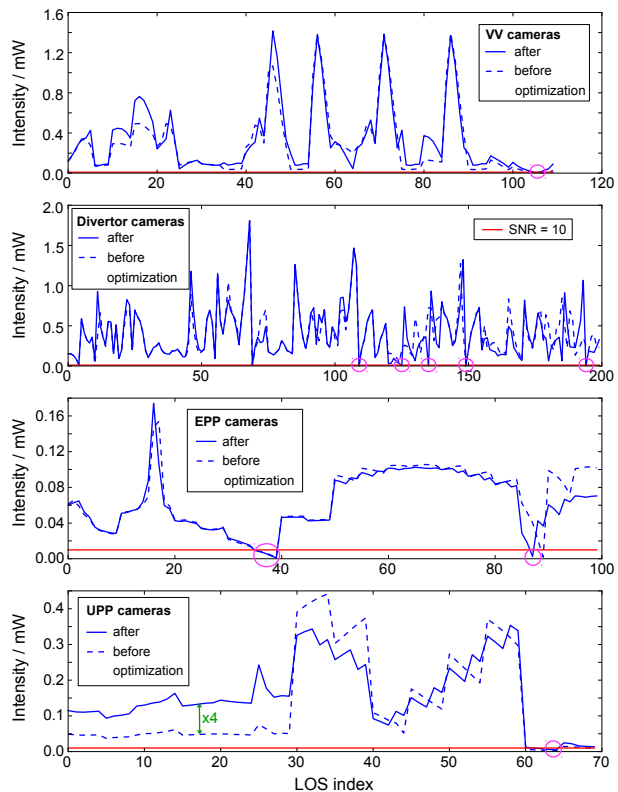

Figure 2: Estimated signal intensities for groups of cameras before and after optimising LOS, given over the index of the LOS within the respective group. LOS indices with $\mathrm{SNR}<10$ after optimisation are marked with a purple circle. (For interpretation of the references to colour in this figure legend, the reader is referred to the web version of this article.)

additional uncertainties it was concluded that the noise level of a bolometer sensor for ITER will be in the range of $1 \mu \mathrm{W}$ detected power, corresponding to a signal of about $20 \mu \mathrm{V}$. This is in line with estimations for the acceptable maximum noise level of $50 \mu \mathrm{V}$, which are based on the requirement for the dynamic range of the diagnostic due to the measurement range to cover. They resulted in a noise budget for signal cables of $0.3-$ $3 \mu \mathrm{V} / \mathrm{m}$ which was achieved by some cable prototypes tested. Bolometer measurements at ASDEX Upgrade and JET also show noise levels in this range [11]. Comparing this to the values of Fig. 2 clearly shows that several LOS are expected to have very low signal levels, partially even a signal-to-noise ratio (SNR) below unity. A closer inspection shows that this applies to those LOS which pass through regions with extremely low radiative power, e.g. the plasma boundary around the upper divertor or at low-field side mid-plane.

\section{Optimisation of LOS}

For increasing the light yield it has to be considered that due to the severe restrictions imposed on the construction space only few improvements to the LOS geometry will be feasible. In particular, the étendue of only few LOS can be increased by widening the corresponding fields of view if in-vessel components in front of bolometer cameras should not vignet the viewing cones.

A possibility to enhance the light yield by a factor of two for all LOS would be to relax the requirement on supressing stray radiation from electron-cyclotron heating. This would open the opportunity to use alternative methods for mm-wave suppression [12] than the microwave reflecting grid. However, the feasibility of this option will have to be determined considering final capabilities for mm-wave suppression and requirements on the suppression factor.

Further improvements of the light yield were identified by re-evaluating the overall distribution of LOS. Those cameras observing the plasma edge with very low intensity should be turned so that fewer LOS of them pass only through the scrape-off layer and thus more LOS receive higher radiative power. Partially, their viewing cone was widened to better match the observed plasma region with those of other cameras. The latter results in a more evenly distributed coverage with slightly increased viewing cones in poloidal direction (and thus higher light yields), but it is achieved on the expense of reduced spatial coverage. Cameras affected by these improvements are mainly those viewing the upper plasma region. The situation before and after redistribution is shown in Fig. 3, left and right, respectively. Camera 1081 was turned slightly more inwards.

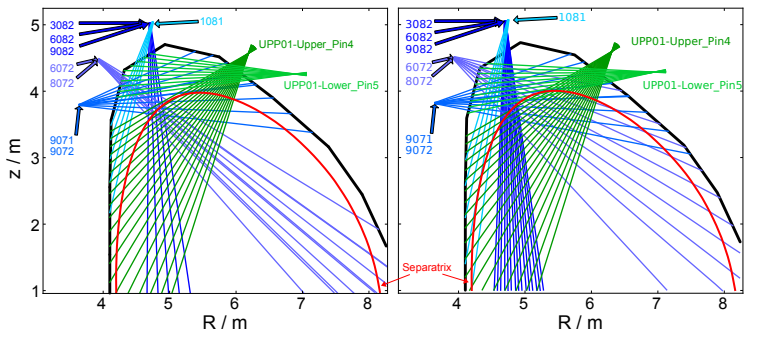

Figure 3: LOS distribution in upper plasma region before (left) and after (right) optimisation procedure.

The viewing cones of the cameras 3082, 6082 and 8082 (observing the divertor region) were widened and interlaced to close the gap in the observation area and enhance spatial resolution. Furthermore, the lower camera in the upper port plug, which observes the upper divertor region, is proposed to be tilted downwards and its viewing cone slightly widened. Finally, the LOS of cameras 6072 and 8072 have been re-distributed to better cover the upper plasma towards the low-field side (LFS). These measures enhance the expected signals as 
demonstrated in Fig. 2, top.

For port mounted cameras the design was optimised to make use of as much width of the viewing cone in toroidal direction as the cutout in BMs allows. However, it needs to be checked, that this does not deteriorate the spatial resolution due to the increased coverage of the viewing cone and the toroidal curvature of field lines. In the worst case (observation at high-field side (HFS) with a major radius of $4.5 \mathrm{~m}$ ) geometrical considerations show that the toroidal extent of the viewing cone should be less than $1.338 \mathrm{~m}$ for the field line curvature in this area not to cover more than $5 \mathrm{~cm}$ in radial direction. This was assured for all LOS for port mounted cameras. Furthermore, viewing cones were checked to assure that there is at least a margin of $1 \mathrm{~mm}$ tolerance for its width when passing through BMs. These changes resulted in an increase of the light yield for collimator cameras in the UPP by a factor of four but also to some reductions for the pinhole cameras from EPP and UPP observing the LFS plasma edge (see Fig. 2, two bottom graphs).

The distribution and the alignment of divertor cameras had to be re-evaluated after the analysis of the available construction space revealed severe constrictions originating from the $\mathrm{RH}$ installation of divertor cassettes. Envelopes for the construction space of cameras were re-defined resulting in 10 typical locations on the divertor cassette. At most eight of these locations can be occupied per cassette due to limitations of the available electrical feedthroughs. The new distribution of LOS proposed focuses on using 20 out of the 40 available 5-channel cameras to cover the regions between strike points and X-point with the desired spatial resolution. The result is shown in Fig. 4 on the left for 10 cameras, the LOS on top and the resulting projectionspace coverage on the bottom. The other ten locations would be installed in other cassettes with slightly tilted viewing cones so that the spatial resolution is enhanced and the requirement on spatial resolution met. Furthermore, two cameras on the divertor cassettes are used to observe the plasma edge on the HFS and the remaining 18 cameras can be distributed to cover further regions of interest (e.g. between X-point and HFS for observation of MARFEs (radiation instabilities) or radiation patterns in high radiative scenarios with varying location [11]), provide LOS to assess potential toroidal asymmetry in the radiation profile as well as to provide redundancy to account for potential failures during life-time. The full picture of the proposed LOS distribution in the divertor is shown in Fig. 4 on the right. However, this distribution needs further refinement once the feasibility of these locations has finally been approved.
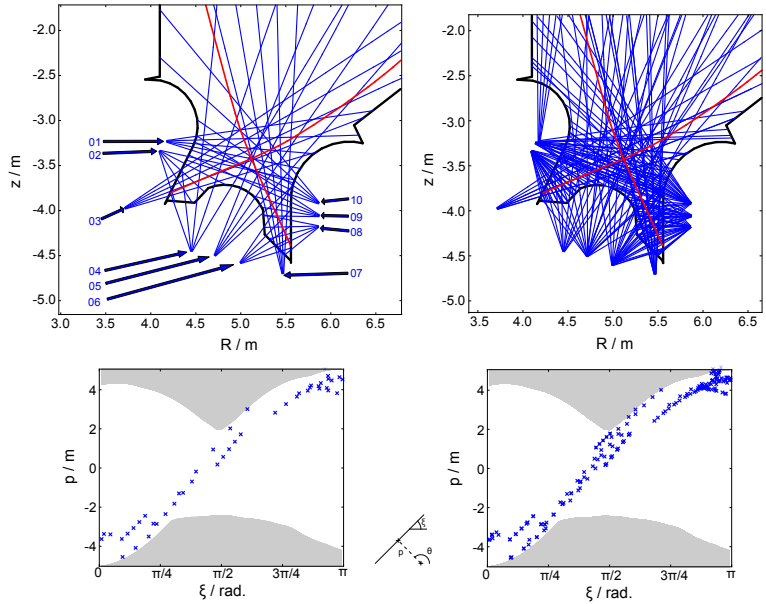

Figure 4: Proposed distribution of LOS in the divertor area. Left for 50 out of 200 LOS indicating the 10 positions (top) and the corresponding projection-space coverage (bottom). Right are all 200 LOS given (top) with the corresponding projection-space coverage (bottom). The definition of the projection space coordinates [6] is illustrated in the sketch (bottom, middle).

Design parameters of the sensors also influence the sensitivity of the bolometer diagnostic. As initial proposals to use Pt as absorber material [13] proved to result in too high intrinsic stresses which lead to mechanical failure of the sensor during thermal cycling [14], ongoing sensor development activities now focus on $\mathrm{Au}$ as absorber material but using Pt for meanders due to the superior temperature coefficient of resistance and reduced neutron cross section. Transmutation of Au over the ITER lifetime is expected to be less than $10 \%$, with very limited impact on spectral response. These time intensive investigations are expected to be concluded within the next three years. For now, this article focuses on a possibility to improve the SNR by tailoring the thickness of the absorber, irrespective of its material.

From the bolometer measurement equation [1] it can be derived, using the assumption of a steady state radiation level, that the measured bridge voltage $U_{b}$ is proportional to the incident radiational power $P_{\text {rad }}$ according to

$$
U_{b} \sim \frac{\tau}{C_{M}} P_{\mathrm{rad}}
$$

with the cooling time constant $\tau$ and the specific heat capacity $C_{M}$ of the absorber. As the spectrum of the radiational power from ITER will extend to energies above $20 \mathrm{keV}$ it is expected that $\mathrm{Au}$ or Pt absorber with thicknesses in the order of $20 \mu \mathrm{m}$ will be required to capture around $90 \%$ of the total radiation. In comparison to absorber thicknesses of 4-8 $\mu \mathrm{m}$ for typical current day sensors this would mean a reduction in sensitivity of a 
factor of 2-5. As LOS in ITER passing only through the plasma edge regions will receive lower maximum photon energies, those LOS could make use of thinner absorbers without reducing their efficiency as they won't receive photons outside of their effective spectral response. Thus they would gain in sensitivity. This optimisation could be applied to all sensors used for the LOS marked in red in Fig. 1.

\section{Conclusion}

The current distribution of the LOS for ITER has been re-visited and assessed in terms of performance. The estimated signal intensities are in the same range as the expected noise levels for those LOS which observe mainly regions of the ITER cross section with very low radiative power densities. Due to the stringent constraints from the available space envelopes for integrating bolometer cameras, the main options for improving the SNR identified are to re-orient few LOS into regions with higher radiative power densities, to widen the apertures of cameras as much as possible and to use different thicknesses of absorbers for LOS observing high and low temperature regions of the plasma. So far, only one radiation profile, evaluated for consistent edge and core radiation, was available for this study. Data for other radiation scenarii, in particular also those for high radiative scenarii and those for the start-up phase of ITER, are currently being calculated. During the ongoing design phase of the ITER bolometers expected signals levels for these scenarii will have to be estimated to predict performance for all phases of ITER operation and to successfully prepare the Preliminary Design Review, which is expected to be held in the first half of 2020. Furthermore, a RAMI analysis needs to be carried out to assess the level of required redundancy. All these considerations are a mandatory input for taking final decisions on the orientation of cameras mounted on the divertor cassettes and the optimised toroidal distribution of LOS as well as for defining the thicknesses of individual bolometer absorbers.

\section{Acknowledgments}

This work was partly supported by Fusion for Energy under the Grant F4E-FPA-384-SG03. The views expressed in this publication are the sole responsibility of the authors and do not necessarily reflect the views of the Fusion for Energy and the ITER Organization. Neither Fusion for Energy nor any person acting on behalf of Fusion for Energy is responsible for the use, which might be made of the information in this publication. The authors greatly acknowledge the work on LOS optimisation of S. Kalvin and A. Suarez on which this effort is based. They also value M. O'Mullane's efforts for providing the synthetic radiation profile (ITER_D_RTP2LV).

\section{References}

[1] K. F. Mast et al. Rev.Sci. Instrum., 62(3), 1991, 744-750.

[2] H. Meister et al. Rev. Sci. Instrum., 79, 2008, 10F511-1 10F511-5, 2008.

[3] A. Pataki et al. Flexible VV bolometer camera design in ITER to adapt to the final position of the gaps between blanket modules. In $30^{\text {th }}$ Symposium on Fusion Technology (SOFT-30), Giardini Naxos, Sicily, Italy, 2018.

[4] A.J.H. Donné et al. Chapter 7: Diagnostics. Nucl. Fusion, 47(6), 2007, S337.

[5] R. Reichle et al. Bolometer for ITER, In: volume 2 of Proceedings of the International Workshop on Diagnostics for ITER, Varenna, Italy, August 28 - September 1, 1995; Plenum Press, $1996,559$.

[6] L.C. Ingesson and R. Reichle. Lines of sight for ITER bolometers. Report jet-r(98)03, 1998, JET Joint Undertaking; Abingdon, Available from http://www.eurofusionscipub.org/archives/jet-archive/lines-of-sight-for-iterbolometers.

[7] H. Meister and S. Kalvin. Fusion Eng. Des., 89(12), 2014, 3039-3045.

[8] D. Vezinet et al. Nuc. Fusion, 56(8), 2016, 086001.

[9] F. Penzel et al. Fusion Eng. Des., 88(6-8), 2013, 1267-1270.

[10] H. Meister et al. Rev. Sci. Instrum., 84(12), 2013.

[11] M. Bernert et al. Nuclear Mater. Energy, 12, 2017, 111 - 118

[12] H. Meister et al. Fusion Eng. Des., 9697, 2015, 861 - 864

[13] H. Meister et al. Rev. Sci. Instrum., 81, 2010, 10E132.

[14] H. Meister et al. Rev. Sci. Instrum., 83, 2012, 10 D724. 